\title{
A blazar as the likely counterpart to 4FGL J0647.7-4418 instead of a gamma-ray binary
}

\author{
Josep Martí, ${ }^{1 \star}$ Estrella Sánchez-Ayaso, ${ }^{2}$ Pedro L. Luque-Escamilla, ${ }^{3}$ Josep M. Paredes ${ }^{4}$, \\ Valentí Bosch-Ramon ${ }^{4}$, and Robin H. D. Corbet ${ }^{5}$ \\ ${ }^{1}$ Departamento de Física (EPSJ), Universidad de Jaén, Campus Las Lagunillas s/n Ed. A3, E-23071 Jaén, Spain \\ 2 Departamento de Ciencias Integradas, Facultad de Ciencias Experimentales, Campus "El Carmen", Universidad de Huelva, Avda. de las Fuerzas \\ Armadas s/n, E-21007 Huelva, Spain \\ ${ }^{3}$ Departamento de Ingeniería Mecánica y Minera (EPSJ), Universidad de Jaén, Campus Las Lagunillas s/n Ed. A3, E-23071 Jaén, Spain \\ ${ }^{4}$ Departament de Física Quàntica i Astrofísica, Institut de Ciències del Cosmos, Universitat de Barcelona, IEEC-UB, Martí $i$ Franquès 1, \\ E-08028 Barcelona, Spain \\ ${ }^{5}$ University of Maryland, Baltimore County, and X-ray Astrophysics Laboratory, Code 662 NASA Goddard Space Flight Center, Greenbelt Rd., \\ $M D$ 20771, USA
}

Accepted XXX. Received YYY; in original form ZZZ

\begin{abstract}
The persistent gamma-ray source 4FGL J0647.7-4418 is tentatively associated in the latest Fermi catalogue with the sub-dwarf O-type X-ray binary HD 49798. However, an AGN candidate is also mentioned as an alternative identification in updated versions of the catalogue accompanying paper. If the first association were correct, this would add HD 49798 to the handful of currently known gamma-ray binaries and, therefore, represent a significant breakthrough not only because of a new member addition, but also because of the apparent white dwarf companion in this system. Despite these perspectives, here we show that the stellar association is likely wrong and that the proposed AGN object, well inside the Fermi $95 \%$ confidence ellipse, is a more conceivable counterpart candidate to the Fermi source due to its strong blazar similarities.
\end{abstract}

Key words: gamma rays: stars - X-rays: binaries - white dwarfs - stars: individual: HD49798 - (galaxies:) BL Lacertae objects: general

\section{INTRODUCTION}

Gamma-ray binaries (GBs) provide one of the most extreme cases of electromagnetic radiative output from gravitationally bound stellar pairs, reaching not only high photon energies (HE, $\sim 1 \mathrm{GeV}$ ), but also very-high-energies (VHE, $\sim 1 \mathrm{TeV}$ ) in some systems. This is a relatively new field in gamma-ray astrophysics that has been intensively reviewed several times in the past years (Dubus 2013, 2015; Paredes \& Bordas 2019b). In particular, high-mass GBs whose optical counterpart is an early-type (O or B) luminous star, with or without emission lines from a circumstellar envelope, are the most distinctive members of the GB family. The taxonomy of stellar gamma-ray sources also includes a variety of other related systems, such as microquasars, colliding wind binaries, novae, and transitional millisecond pulsars (Paredes \& Bordas 2019a), but not often as luminous and persistent as the 'classical' GBs referred above. Room for other kinds of

^ E-mail: jmarti@ujaen.es unexpected gamma-ray stellar emitters could also await discovery.

The nature of the unseen companion in the most common case of high-mass GBs is still under an animated debate. The exception is in the case where pulsations have been detected and attributed to a neutron star whose rotational energy is suspected to act as the ultimate power source. High-mass GBs typically display both HE and VHE emission modulated with the system orbital, and sometimes also super-orbital, period(s). Therefore, the physical conditions for acceleration of particles and different emission processes repeat in a predictable way that facilitates their study. The wide attention raised by this special kind of systems is in contrast with the reduced number currently being known as only $\sim 10$ sources have been detected so far. Moreover, the perspectives for new Milky Way discoveries do not appear to be very optimistic based on a population synthesis analysis that estimated a total number of only $\sim 10^{2}$ systems in our Galaxy (Dubus et al. 2017).

The GB scarcity contrasts with the dominant abun- 
dance of blazar-type sources across the whole sky in the present and past Fermi catalogue series. The latest of these, nicknamed as 4FGL, was released after 8 year of data collection with the Large Area Telescope (LAT) on board NASA's Fermi space observatory (The Fermi-LAT collaboration 2019). Remarkably, this catalogue proposes the association of one of its persistent sources, namely 4FGL J0647.7-4418, with the already known X-ray binary 1WGA J0648.0-4419 (Liu et al. 2006). This fact immediately prompted us to explore in more detail the chances of a real association with the gamma-ray source as suggested by the Fermi catalogue itself. Several of the currently recognized GBs were previously known as common X-ray binaries and we wondered if HD 49798 could follow the same path. A real association would not only mean incremental progress in the field, but also a class-broadening of the GB family since its compact object is more consistent with a spun-up white dwarf rather than a neutron star.

At this point we must indicate that, starting with the $3^{r d}$ draft version of the 4 FGL accompanying paper, posted by the Fermi collaboration, an important information with respect to this binary system was added. The Fermi team noticed that the binary association probability (85\%) was barely larger than that of a nearby blazar candidate (80\%) not considered before. Despite this, the latest electronic version of the 4FGL catalogue still lists the binary association as the only one and 4FGL J0647.7-4418 remains classified as a high mass binary. In this paper, we provide significant evidence indicating that this classification is likely wrong and in need of revision.

\section{THE GB SUSPECT}

HD 49798 is a bright $(V=8.27)$, early-type star HD 49798 located well below the Galactic plane $\left(l^{I I}=253.71, b^{I I}=\right.$ $-19.14)$, and at a distance of $508 \pm 16$ pc according to the Gaia Data Release 2 (Gaia Collaboration et al. 2018). Its peculiar nature was originally pointed out by the renowned Jascheck spectroscopists, who recognized it as a hot subdwarf of O6 spectral type (sdO6, luminosity class VI) using plates taken at the Bosque Alegre station of Córdoba Observatory in Argentina (Jaschek \& Jaschek 1963). Early-type, hot subdwarfs are believed to originate when a red giant star gets rid of its outer layers of hydrogen before the core starts to fuse helium.

The Jaschecks work was followed by a spectroscopic orbital solution that yielded an orbital period of $1.55 \mathrm{~d}$ and mass function of about $0.27 M_{\odot}$ (Thackeray 1970), values later confirmed using IUE spectra (Stickland \& Lloyd 1994). Hints about the nature of the HD 49798 companion emerged finally thanks to ROSAT X-ray observations (Israel et al. 1997), that revealed the existence of a pulsation period of $P=13.2$ s attributed to either a white dwarf (WD) or a magnetic neutron star (NS). The discovery with XMM-Newton of X-ray eclipses allowed to highly constrain the system orbital inclination (Mereghetti et al. 2011). These authors also combined this information with the $\mathrm{X}$-ray pulse delays and the optical mass function. In this way, it was possible to fully solve for the mass of the sub-dwarf $\operatorname{star}\left(M_{*}=1.50\right.$ $\left.M_{\odot}\right)$ and that of the compact object $\left(M_{X}=1.28 M_{\odot}\right)$. With this information in hand, the classification of HD 49798 as a

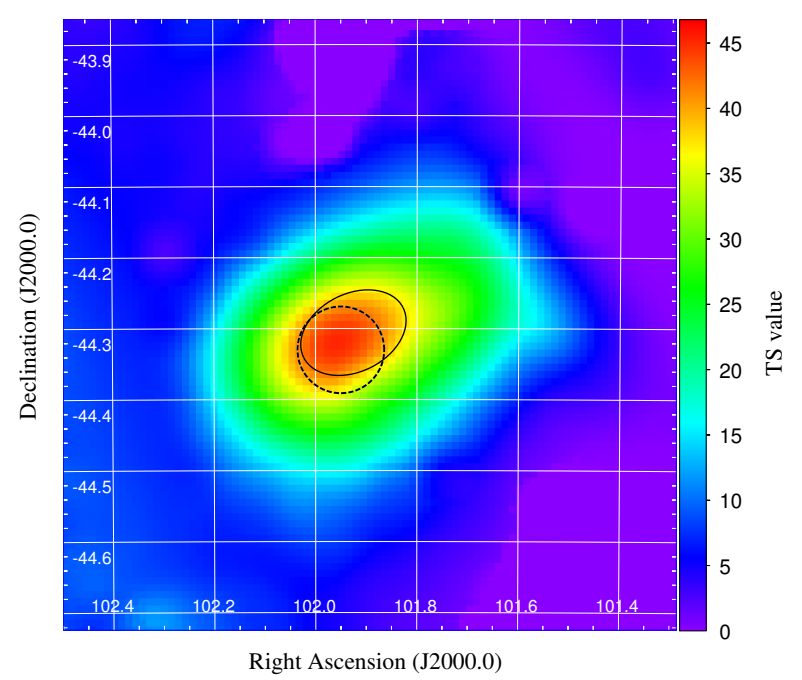

Figure 1. TS map $\left(1^{\circ} \times 1^{\circ}\right)$ of the 4 FGL J0647.7-4418 region resulting from our own analysis of Fermi-LAT data. The black dashed circle represents the improved 95\% confidence level gamma-ray source location. The black ellipse corresponds to the same confidence level location according to the 4FGL catalogue.

high-mass X-ray binary becomes mostly based on the spectral type earliness of the donor star rather than its mass.

A latter X-ray analysis by Mereghetti et al. (2016) remarkably revealed a steady spin-up of the pulsed emission of $\dot{P}=-2.15 \times 10^{-15} \mathrm{~s} \mathrm{~s}^{-1}$, initially attributed to accretion of the wind from the sub-dwarf companion onto a NS. However, the observed spin-up stability over 20 years is very unusual in NS X-ray binaries. Recently, this effect has been reinterpreted in a consistent WD context under contraction because of its young age $(\sim 2 \mathrm{Myr})$, with the current radius estimate being $R_{X} \simeq 3340 \mathrm{~km}$ (Popov et al. 2018). The spin-up contribution due to stellar wind accretion from the sub-dwarf, with mass loss $\dot{M}_{w}=3 \times 10^{-9} M_{\odot} \mathrm{yr}^{-1}$ and terminal velocity $v_{\infty} \simeq 1350 \mathrm{~km} \mathrm{~s}^{-1}$ (Hamann 2010), is found to be negligible in this case. Independently of the compact object nature, the physical properties of the HD 49798 system strongly depart from a typical high mass X-ray binary. How HD 49798 could fit into the GB family, where no sub-dwarfs are found and no clear scenario for gamma-ray emission is available, started to be challenging.

\section{RE-ANALYSIS OF $F E R M I$-LAT DATA}

Our first step was to perform a brief re-analysis of the FermiLAT Pass 8 database towards HD 49798, which included data from August 4, 2008 (MET 239557417) until July 1, 2019 (MET 583643061). The immediate purpose was to explore if some improvement with respect to the 4FGL catalogue was achievable, specially looking forward to a more accurate gamma-ray source location. We selected the front and back converted photons at energies from $100 \mathrm{MeV}$ to 1 $\mathrm{TeV}$ within a $5^{\circ} \times 5^{\circ}$ square region centered at the position of the source, considered as the region of interest (ROI). In order to exclude time periods in which some spacecraft event had affected the data quality, we used the source event class recommended for individual source analysis using the ex- 
pression (DATA_QUAL $>0) \& \&($ LAT_CONFIG $==1)$. Moreover, only events with zenith angles lower than $90^{\circ}$ were taken into account to reduce the background contamination from the Earth's albedo. We used the current Fermitools from conda distribution and the latest version of the instrument response functions (IRFs) P8R3_SOURCE_V2 to process our data. All steps included in the Fermitools tutorial ${ }^{1}$ for unbinned likelihood analysis were carefully followed. We also ran the make4FGLxml.py script ${ }^{2}$ within the ROI, employing the 4FGL catalogue and making use of the Galactic diffuse model gll_iem_v07.fits and isotropic emission model iso_P8R3_SOURCE_V2_v1.txt ${ }^{3}$.

As a result of our re-analysis we obtained a detection very compatible with the one provided by the 4FGL catalogue, with just a slightly fainter integral photon flux of $(2.0 \pm 0.4) \times 10^{-10}$ photon $\mathrm{cm}^{-2} \mathrm{~s}^{-1}$ from 1 to $100 \mathrm{GeV}$. The resulting spectrum was adequately described by a simple power-law type $d N / d E=N_{0}\left(E / E_{0}\right)^{-\gamma}$ with index $\gamma=2.2 \pm 0.2$. The corresponding Test Statistic (TS) value was 47 , which translated into a detection significance of about 7 . This confirmed the reality of 4FGL J0647.7-4418 with two additional years of data not included in the 4FGL catalogue.

The resulting TS map is presented in Fig. 1. Our best estimate for the J2000.0 source position (Right Ascension = 101.9500; Declination $=-44.3300$ ), with error radius 0.06 at the $95 \%$ confidence level, was finally derived from it by using the task gtfindsource and the plot_tsmap.py script ${ }^{4}$.

\section{LOOKING FOR THE ORBITAL PERIOD SIGNATURE IN FERMI DATA}

The light curve of Fermi-LAT data was also extracted around the HD 49798 position in order to search its power spectrum for the known orbital period of the system. We used data covering the interval from MJD 54682 to 58745 with a time resolution of $500 \mathrm{~s}$. The technique of probabilityweighted aperture photometry was used for this purpose. This is the same technique that led to the most recent discoveries of the GBs LMC P3 (Corbet et al. 2016) and 4FGL J1405.1-6119 (Corbet et al. 2019). The resulting power spectrum is shown in Fig. 2. When calculating it, weights were given according to the relative exposure of each data point.

The power spectrum displayed in Fig. 2 does not show any significant power component matching the HD 49798 orbital cycle. This negative result is relevant since one might expect to see some signal at the orbital period value if the binary system was the true source of HE photons.

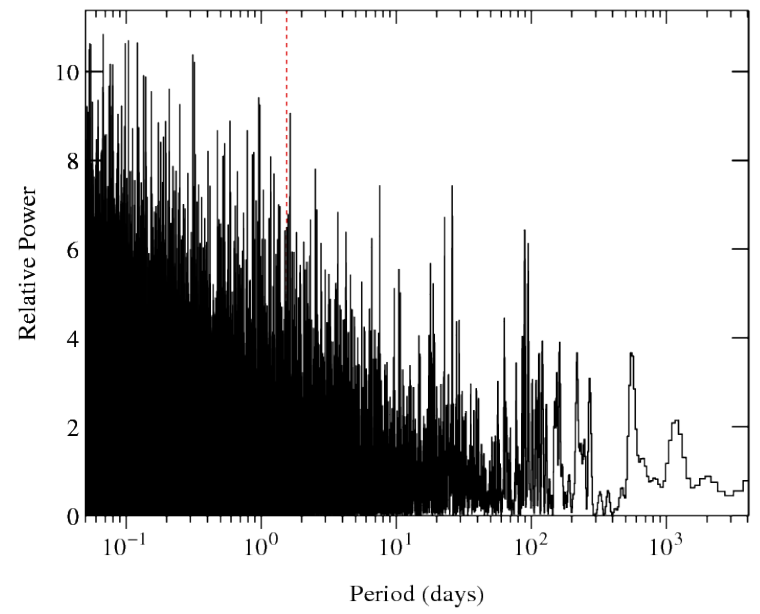

Figure 2. Power spectrum of the Fermi-LAT light curve computed as described in the text. The dashed red line indicates the location of the most accurate orbital period of $1.547666 \mathrm{~d}$ (Mereghetti et al. 2016) where no relevant power is seen.

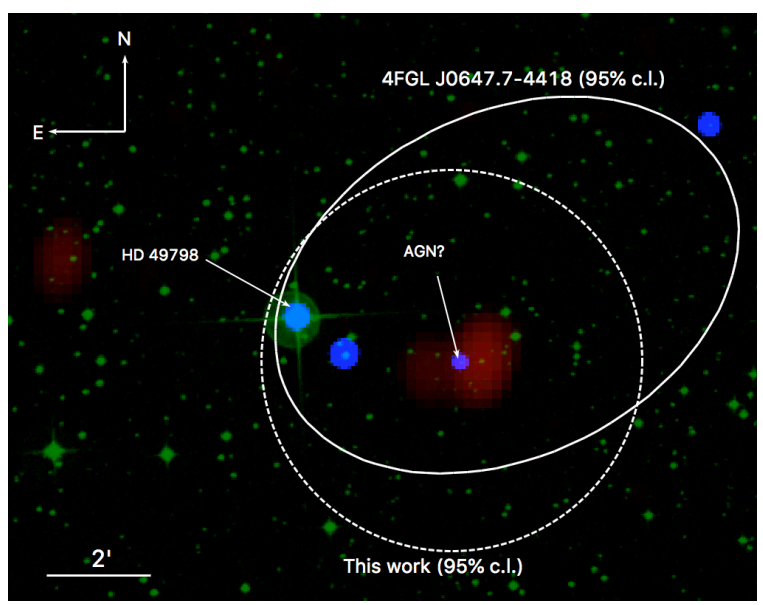

Figure 3. Tri-chromatic view of the region of the 4 FGL J0647.7-4418 confidence ellipse at the 95\% level where the red, green and blue layers correspond to radio, optical and X-ray emission downloaded from the SUMSS $843 \mathrm{MHz}$, DSS2 blue plate and XMM-Newton archives, respectively. The improved $95 \%$ confidence position resulting from our own analysis of Fermi data is also included as a dashed circle. The location of HD 49798 and the suspected AGN source are arrowed on the image.

\section{MULTI-WAVELENGTH SEARCH FOR ALTERNATIVE COUNTERPARTS}

In Fig. 3, a tri-chromatic view of the 4FGL J0647.7-4418/HD49798 field is shown with the $95 \%$

\footnotetext{
1 https://fermi.gsfc.nasa.gov/ssc/data/analysis/scitools/ likelihood_tutorial.html

2 https://fermi.gsfc.nasa.gov/ssc/data/analysis/user/ make4FGLxml.py

3 https://fermi.gsfc.nasa.gov/ssc/data/access/lat/BackgroundModels.html

4 http://grbworkshop.wikidot.com/s6-fermi-lat-analysis-handson
} 
Table 1. X-ray sources consistent with 4FGL J0647.7-4418

\begin{tabular}{|c|c|c|c|c|c|c|c|c|}
\hline \multirow[b]{2}{*}{$3 \mathrm{XMM}$} & \multicolumn{3}{|c|}{ Source location } & \multicolumn{3}{|c|}{ Flux $\left(10^{-14} \mathrm{erg} \mathrm{cm}^{-2} \mathrm{~s}^{-1}\right)$} & \multirow{2}{*}{$\begin{array}{l}\text { Hardness } \\
\text { ratio } \\
\frac{F_{\text {hard }}-F \text { soft }}{F_{\text {total }}}\end{array}$} & \multirow{2}{*}{$\begin{array}{c}\text { Optical } \\
\text { counterpart }\end{array}$} \\
\hline & $\begin{array}{l}\text { R. A. } \\
\left({ }^{\circ}\right)\end{array}$ & $\begin{array}{l}\text { Dec. } \\
\left({ }^{\circ}\right)\end{array}$ & $\begin{array}{l}\text { Pos. Err } \\
\text { (") }\end{array}$ & $\begin{array}{c}F_{\text {total }} \\
0.5-12 \mathrm{keV}\end{array}$ & $\begin{array}{c}F_{\text {soft }} \\
0.5-2 \mathrm{keV}\end{array}$ & $\begin{array}{c}F_{\text {hard }} \\
2-12 \mathrm{keV}\end{array}$ & & \\
\hline J064747.1-441950 & 101.94638 & -44.33063 & 0.11 & 15.6 & 5.0 & 10.6 & +0.36 & $\mathrm{AGN}^{a}$ \\
\hline J064759.5-441941 & 101.99824 & -44.32828 & 0.09 & 18.6 & 16.8 & 1.7 & -0.81 & $\mathrm{Star}^{b, c}$ \\
\hline J064804.6-441858 ${ }^{d}$ & 102.01953 & -44.31623 & 0.09 & 13.7 & 5.3 & 8.4 & +0.23 & HD 49798 \\
\hline
\end{tabular}

${ }^{a}$ Candidate AGN classification (Lin et al. 2012). ${ }^{b}$ Candidate star classification (Lin et al. 2012). ${ }^{c}$ Significant parallax and proper motion available (Gaia Collaboration et al. 2018). ${ }^{d}$ Also known as RX J0648.0-4418.

confidence regions of 4FGL J0647.7-4418 from both the Fermi-LAT catalogue and our own work over-plotted onto it. The blue layer corresponds to X-ray emission as observed with the XMM-Newton observatory where, according to the 3XMM-DR8 catalog release ${ }^{5}$ (Zolotukhin et al. 2017), HD 49798 agrees within $\sim 0.1$ arcsecond with the bright X-ray source 3XMM J064804.6-441858. The green layer represents the optical appearance of the field as retrieved from the digitized version of the second Palomar Observatory Sky Survey (POSS II), with the blue plate being used (Reid et al. 1991). Finally, the red layer displays radio emission at $843 \mathrm{MHz}$ from the Sydney University Molonglo Sky Survey (SUMSS) (Mauch et al. 2003).

Table 1 contains the relevant information for all the three X-ray sources consistent with the Fermi source sorted by right ascension. The first of them is 3XMM J064747.1-441950, which has been proposed as a possible Active Galactic Nucleus (AGN) in Lin et al. (2012) and will be considered in more detail below. The second one is 3XMM J064759.5-441941, which agrees within 0.2 arcsecond with an anonymous stellar object. According to the Gaia Data Release 2 (Gaia Collaboration et al. 2018), both a high parallax (10.74 milli-arcsecond) and proper motion (10.4 milli-arcsecond year $^{-1}$ ) are reported for this object, therefore ensuring its stellar nature. Assuming negligible reddening because of its proximity, the observed brightness level $(G=12.88)$ and low effective temperature $\left(T_{\mathrm{eff}}=4100\right.$ $\mathrm{K})$ points to a main sequence star of M-type with chromospheric activity unlikely to produce detectable gamma-rays. The third and last source is 3XMM 064804.6-441858, the previously mentioned X-ray counterpart of HD 49798 that motivated our work.

\section{A BLAZAR AGN COUNTERPART}

At first glance, the candidate AGN 3XMM J064747.1-441950 within the 4FGL J0647.7-4418 location does not seem to match any of the radio sources in the field according to the SUMSS (red layer in Fig. 3). We also checked this using the $150 \mathrm{MHz}$ data from the TIFR GMRT Sky Survey (TGSS), with slightly better angular resolution but less sensitivity, as provided by its first alternative data release (Intema et al. 2017). The closest SUMSS radio source is coincident with TGSS J064744.7-441947, offset by about $24^{\prime \prime}$ approximately to the West. It is worth to mention here the existence of a second fainter SUMSS

\footnotetext{
5 http://xmm-catalog.irap.omp.eu
}

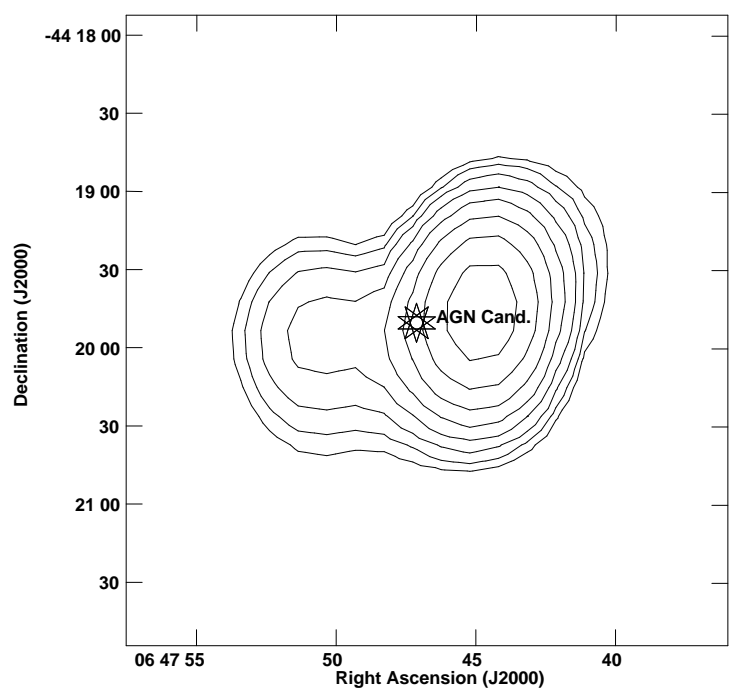

Figure 4. Zoomed countour map of SUMSS radio emission at $843 \mathrm{MHz}$ towards the X-ray candidate AGN 3XMM J064747.1-441950, whose position is marked by the star symbol. Contours show start at four times the rms noise of $1.3 \mathrm{mJy} /$ beam and proceed in $\sqrt{2}$ steps.

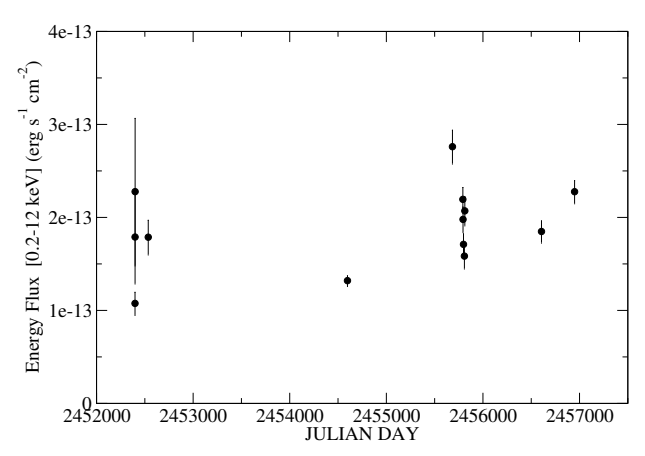

Figure 5. Archival X-ray light curve of the candidate AGN 3XMM J064747.1-441950 in the energy range 0.2-12 keV. 


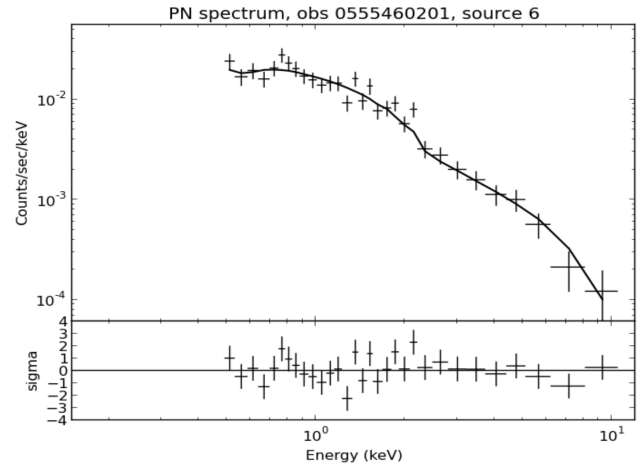

Figure 6. Archival spectrum of the candidate AGN 3XMM J064747.1-441950 as observed with the EPIC pn cameras on board the XMM-Newton observatory during a 42 ks exposure (date 5th October 2008).

detection, absent in the TGSS, located about 40" away to the East and with a roughly opposite position angle with respect to the proposed AGN. This relative position is morphologically reminiscent of a pair of extended radio lobes as displayed in the Fig. 4 zoomed contour plot. Despite the poor angular resolution of the survey, the $\sim 4$ brightness contrast between the two apparent lobes is suggestive of possible Doppler-boosted jets emanating from 3XMM J064747.1-441950, which is located just in between. Moreover, it is worth to mention that when the SUMSS flux density of the brightest radio source is combined with its TGSS measurement, a tentative non-thermal spectral index $\alpha \simeq-0.6 \pm 0.3$ is obtained assuming constant emission.

Retrieving flux measurements from the XMM-Newton Survey Science Centre ${ }^{6}$, 3XMM J064747.1-441950 appears as a persistent X-ray source over time scales of years. In Fig. 5, we compiled the available X-ray light curve in the $0.2-12 \mathrm{keV}$ range. The source is clearly variable by a factor of $\sim 2$ on time scales of at least months. Unfortunately, its time sampling is not dense enough for a meaningful comparison with Fermi LAT data. When observed with the longest on-source time (see Fig. 6), the source X-ray spectrum was better fitted by a simple absorbed power-law with photon index $1.8 \pm 0.1$ and hydrogen column density $(8 \pm 1) \times 10^{20}$ $\mathrm{cm}^{-2}$. The corresponding reduced $\chi^{2}$ value amounted to 1.1 over 28 degrees of freedom.

\subsection{Photometric support for a blazar identification}

Further inspection of POSS II plates additionally reveals a faint optical source whose position is in subarsecond agreement with the 'central' $\mathrm{X}$-ray source $3 \mathrm{XMM}$ J064747.1-441950. Shining at $R \simeq 19.0$, this point-like object corresponds to entry \# 0456-0065720 in the USNO_B1_0 Catalog (Monet et al. 2003), where its lack of proper motion also agrees with an extragalactic origin. In the absence of spectroscopic information, a reassuring information about the nature of this AGN candidate comes from the infrared

${ }^{6}$ http://xmm-catalog.irap.omp.eu

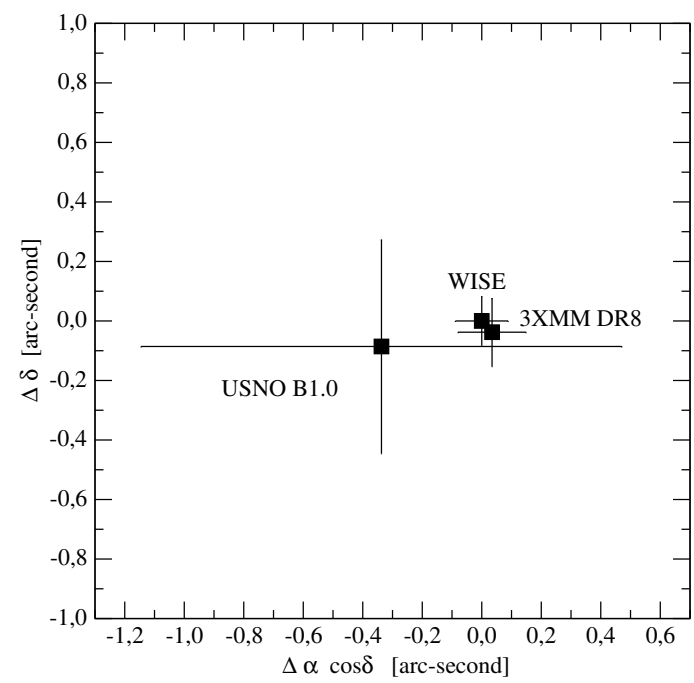

Figure 7. Relative positions of the X-ray source 3XMM J064747.1-441950, the optical source USNO_B1_0 \# 04560065720, and the infrared source AllWISE J064747.12-441950.3, which we propose to originate from the same object.

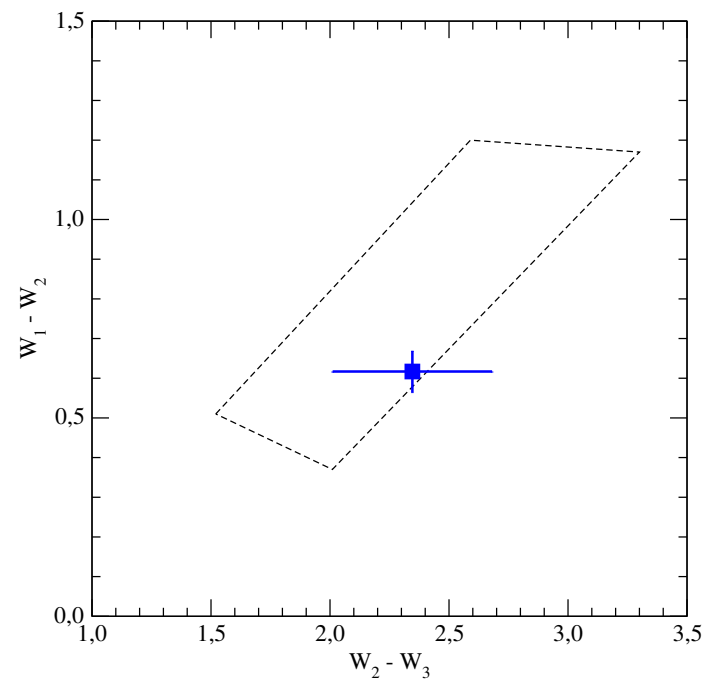

Figure 8. Colour-colour diagram showing the WISE Blazar Strip limits as dashed lines and the location of the WISE source J064747.12-441950.3 in blue.

domain. This is thanks to the Wide-field Infrared Survey Explorer (WISE) and its associated catalogue releases (Wright et al. 2010; Cutri \& et al. 2013), where we find the AllWISE J064747.12-441950.3 source. To confirm as confidently as possible that the XMM, USNO and this WISE object are actually the same, we plot their relative positions in Fig. 7 where their mutual match agrees within a fraction of an arc-second. 
The WISE catalogue series enabled the development of a powerful photometric tool to identify blazar AGNs within the error box of Fermi unassociated sources. We refer here to the WISE Blazar Strip technique developed some years ago and successfully put to test in recent times (Massaro et al. 2012; Massaro \& D'Abrusco 2016). These authors discovered that non-thermal emission in blazars clusters them in a narrow strip of the WISE colour-colour diagram, thus significantly facilitating their identification.

In our case, the WISE source magnitudes in the $3.4 \mu \mathrm{m}$, $4.6 \mu \mathrm{m}$ and $11.6 \mu \mathrm{m}$ relevant bands are $W_{1}=15.445 \pm 0.037$, $W_{2}=14.828 \pm 0.052$ and $W_{3}=12.482 \pm 0.331$, respectively. The corresponding colours have been plotted in Fig. 8 together with the accepted WISE Blazar Strip limits. The agreement is remarkable and we believe this has to be taken as an indication that 4FGL J0647.7-4418 is more likely to be a blazar than associated with the X-ray binary HD 49798. Moreover, in Fig. 5 of Massaro \& D'Abrusco (2016), a strong correlation between the Fermi spectral index and the $W_{1}-W_{2}$ colour was also established for blazars. Plotting our source onto it (not shown here) gives further support to the proposed blazar.

\subsection{A blazar-like spectral energy distribution}

In an attempt to further characterize the nature of our blazar candidate, we have assembled in Figure 9 a tentative spectral energy distribution (SED). This plot combines the multiwavelength data from the counterparts attributed in previous sections to 4FGL J0647.7-4418 at lower energies (radio, infrared, optical and X-rays). The Galactic absorption correction was estimated using NASA's HEASARC tool for hydrogen column density estimates ${ }^{7}$. This tool provided an average value of $(6 \pm 2) \times 10^{20} \mathrm{~cm}^{-2}$ within 0.5 degrees around the target. We used this number, together with the Morrison \& McCammon (1983) cross sections, to place X-ray flux measurements in the XMM-Newton bands into the SED plot in terms of unabsorbed flux per unit of frequency and times the frequency. The corresponding optical extinction turns out to be very low $\left(A_{R} \simeq 0.2 \mathrm{mag}\right)$ and practically negligible at infrared wavelengths.

The resulting SED overall appearance is strongly reminiscent of blazar sources. The available data points, although scarce, clearly outline the existence of the two peaks of synchrotron and inverse Compton origin typically displayed by blazar SEDs. To narrow the possible type of blazar powering the 4FGL J0647.7-4418 emission, we tried to fit the SED points using the Paiano et al. (2017) approach to unveil blazars among multi-wavelength counterparts of Fermi unassociated sources. Their parametrised SED fits use an analytic expression for the blazar luminosity $L_{v}$. We adapt it here to the monochromatic flux $F_{v}$ as a function of frequency $v$ as follows:

$v F_{v}=v F_{1}(v)+v F_{2}(v)$

where

$v F_{i}(v)=A_{i}\left(\frac{v}{v_{i}}\right)^{1-\alpha} \exp \left\{-\frac{1}{2 \sigma_{i}^{2}}\left[\log \left(1+\frac{v}{v_{i}}\right)\right]^{2}\right\}$

7 https://heasarc.gsfc.nasa.gov/cgi-bin/Tools/w3nh/w3nh.pl

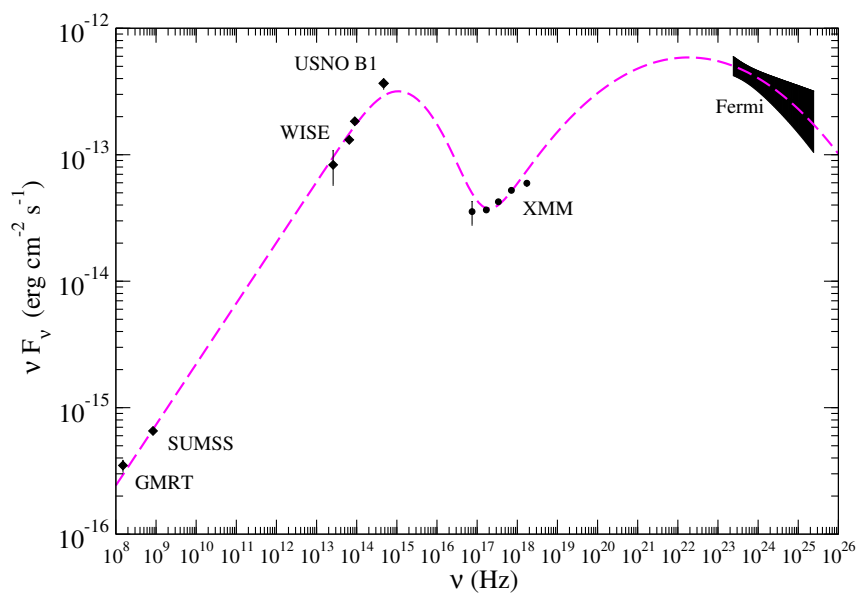

Figure 9. Tentative SED of the proposed blazar source 4FGL J0647.7-4418. The magenta dashed line is a fitting attempt using the analytic expression by Paiano et al. (2017) and the parameters quoted in the text.

represents the synchrotron $(i=1)$ and inverse Compton $(i=$ 2) peaks, respectively. The seven parameters of Eq. 1 include $A_{1}$ and $A_{2}$ for normalization, the two frequency bumps $v_{1}$ and $v_{2}$, their widths $\sigma_{1}$ and $\sigma_{2}$, and a common spectral index $\alpha$.

Unfortunately, we cannot introduce any redshift correction because spectroscopic observations are not available and we are forced to proceed assumig $z=0$. Moreover, the SED data available provides an insufficient sampling of the frequency space to enable the convergence of a full simultaneous least squares fit. To mitigate this added problem, we started by estimating the spectral index with a simple power-law fit restricted to the low-frequency region of radio and infrared data. The result was $\alpha=0.52 \pm 0.01$ and this parameter was kept fixed at all times. Later we proceeded to solve iteratively for each of the rest of parameters under a least squares criterion. The most plausible fit was obtained for $A_{1}=(2.1 \pm 0.6) \times 10^{-13} \mathrm{erg} \mathrm{s}^{-1} \mathrm{~cm}^{-2}, A_{2}=(5.2 \pm 0.2) \times 10^{-14}$ $\mathrm{erg} \mathrm{s}^{-1} \mathrm{~cm}^{-2}, \sigma_{1}=0.9 \pm 0.1, \sigma_{2}=2.0 \pm 0.2, v_{1}=(1.3 \pm 0.6) \times 10^{14}$ $\mathrm{Hz}$, and $v_{2}=(7.6 \pm 0.3) \times 10^{17} \mathrm{~Hz}$.

Despite the SED limited sampling, both in frequency and time, our best guess for the $v_{1}$ parameter suggests that we are dealing with a Low-Synchrotron-Peaked (LSP), or perhaps an Intermediate-Synchrotron-Peaked (ISP), blazar. The distinction between the two classes is arbitrarily set for $v_{1}$ below or above $10^{14} \mathrm{~Hz}$ (Ackermann et al. 2015) and we are just in the transition region.

The classification of blazars is also possible using flux ratios, or broad band spectral indices. From our SED analysis, we find the following approximate flux ratios between radio, X-rays and gamma-rays: $\log \left(F_{\text {radio }} / F_{\mathrm{X}}\right) \simeq 6.9$ and $\log \left(F_{\text {gamma }} / F_{\mathrm{X}}\right) \simeq-5.8$. The corresponding spectral indices are $\alpha_{\text {radio-X }} \simeq 0.80$ and $\alpha_{\mathrm{X} \text {-gamma }} \simeq 0.87$, with the reference frequencies being set to $1.4 \mathrm{GHz}, 2 \mathrm{keV}$ and $10 \mathrm{GeV}$. Bringing these values into the classification plots of Paiano et al. (2017) Fig. 26 (not shown here), they appear to be also lo- 
cated in the region in between LSP and ISP blazars, thus supporting our preliminary classification.

\section{CONCLUSIONS}

The main conclusion of this work is a strong word of caution concerning the association of the gamma-ray source 4FGL J0647.7-4418 with the O-type X-ray binary HD 49798 as proposed in the latest Fermi catalogue.

In addition, an alternative counterpart candidate of likely blazar nature, the X-ray source 3XMM J064747.1-441950, almost centered inside the Fermi LAT 95\% confidence ellipse, is proposed as a more reliable identification. This statement is mainly supported by: i) the existence of non-thermal radio emission symmetrically located around it (SUMSS and GMRT sources); ii) the non-detection of the binary orbital period in the Fermi LAT light curve; iii) the agreement of the object's infrared colours with the well established WISE Blazar Strip; iv) the similarity of the double-peaked SED with that of LSP/ISP blazars; v) the agreement of radio/X-ray/gamma flux ratios and spectral indices also with LSP/ISP blazars. Taken together, all these facts would render 4FGL J0647.7-4418 a very ordinary blazar emitter of gamma rays instead of a new addition to the scarce family of GBs.

\section{ACKNOWLEDGEMENTS}

This work was supported by the Agencia Estatal de Investigación grants AYA2016-76012-C3-1-P and AYA2016-76012C3-3-P from the Spanish Ministerio de Economía y Competitividad (MINECO), by Consejería de Economía, Innovación, Ciencia y Empleo of Junta de Andalucía under research group FQM-322, by grant MDM-2014-0369 of the ICCUB (Unidad de Excelencia 'María de Maeztu'), and by the Catalan DEC grant 2017 SGR 643, as well as FEDER funds. RC acknowledges support from NASA Fermi grant NNX15AU83G.

\section{REFERENCES}

Ackermann M., et al., 2015, ApJ, 810, 14

Corbet R. H. D., et al., 2016, ApJ, 829, 105

Corbet R. H. D., et al., 2019, ApJ, 884, 93

Cutri R. M., et al. 2013, VizieR Online Data Catalog, 2328

Dubus G., 2013, A\&ARv, 21, 64

Dubus G., 2015, Comptes Rendus Physique, 16, 661

Dubus G., Guillard N., Petrucci P.-O., Martin P., 2017, A\&A, 608, A59

Gaia Collaboration et al., 2018, A\&A, 616, A1

Hamann W.-R., 2010, Ap\&SS, 329, 151

Intema H. T., Jagannathan P., Mooley K. P., Frail D. A., 2017, A\&A, 598, A78

Israel G. L., Stella L., Angelini L., White N. E., Kallman T. R., Giommi P., Treves A., 1997, ApJ, 474, L53

Jaschek M., Jaschek C., 1963, Publications of the Astronomical Society of the Pacific, 75, 365

Lin D., Webb N. A., Barret D., 2012, ApJ, 756, 27

Liu Q. Z., van Paradijs J., van den Heuvel E. P. J., 2006, A\&A, 455,1165

Massaro F., D’Abrusco R., 2016, ApJ, 827, 67
Massaro F., D'Abrusco R., Tosti G., Ajello M., Gasparrini D., Grindlay J. E., Smith H. A., 2012, ApJ, 750, 138

Mauch T., Murphy T., Buttery H. J., Curran J., Hunstead R. W., Piestrzynski B., Robertson J. G., Sadler E. M., 2003, MNRAS, 342,1117

Mereghetti S., La Palombara N., Tiengo A., Pizzolato F., Esposito P., Woudt P. A., Israel G. L., Stella L., 2011, ApJ, 737, 51

Mereghetti S., Pintore F., Esposito P., La Palombara N., Tiengo A., Israel G. L., Stella L., 2016, MNRAS, 458, 3523

Monet D. G., et al., 2003, AJ, 125, 984

Morrison R., McCammon D., 1983, ApJ, 270, 119

Paiano S., Franceschini A., Stamerra A., 2017, MNRAS, 468, 4902

Paredes J. M., Bordas P., 2019a, Rendiconti Lincei. Scienze Fisiche e Naturali, p. 11

Paredes J. M., Bordas P., 2019b, arXiv e-prints, p. arXiv:1901.03624

Popov S. B., Mereghetti S., Blinnikov S. I., Kuranov A. G., Yungelson L. R., 2018, MNRAS, 474, 2750

Reid I. N., et al., 1991, PASP, 103, 661

Stickland D. J., Lloyd C., 1994, The Observatory, 114, 41

Thackeray A. D., 1970, MNRAS, 150, 215

The Fermi-LAT collaboration 2019, arXiv e-prints, p. arXiv: 1902.10045

Wright E. L., et al., 2010, AJ, 140, 1868

Zolotukhin I. Y., Bachetti M., Sartore N., Chilingarian I. V., Webb N. A., 2017, ApJ, 839, 125

This paper has been typeset from a $\mathrm{T}_{\mathrm{E}} \mathrm{X} / \mathrm{LAT}_{\mathrm{E}} \mathrm{X}$ file prepared by the author. 